\title{
Expression of the B-Cell Proliferation Marker MUM1 by Melanocytic Lesions and Comparison with S100, gp100 (HMB45), and MelanA
}

\author{
Uma Sundram, M.D., Ph.D., Jeff D. Harvell, M.D., Robert V. Rouse, M.D., \\ Yasodha Natkunam, M.D., Ph.D. \\ Department of Pathology, Stanford University Medical Center, Stanford, California
}

The diagnosis of malignant melanoma remains one of the most difficult to render in surgical pathology, partially because of its extreme histologic variability. Limits in the sensitivity and/or specificity of the currently available melanocytic markers such as anti-S100, HMB45, and anti-MelanA further complicate this problem. Previous work has demonstrated that the B-cell proliferation/differentiation marker MUM1/IRF4 is detected in malignant melanoma and hematolymphoid malignancies, but not in any other neoplasm tested (including colonic, lung, breast, and ovarian carcinomas). In the current study, we have examined MUM1 protein expression in 61 melanocytic lesions and compared the diagnostic usefulness of this marker with that of anti-S100, HMB45, and anti-MelanA. The results indicate that MUM1 is positive in 33/36 (92\%) cases of melanoma (21/22 [95\%] conventional primary melanomas and 12/14 [86\%] metastatic melanomas). In comparison, positivity was seen with anti-S100 in $36 / 36$ cases $(100 \%, 22$ primary and 14 metastatic), HMB45 in 28 cases $(78 \%, 17$ primary and 11 metastatic), and anti-MelanA in 27 cases $(75 \%, 19$ primary and 8 metastatic). Although negative in schwannomas, neurofibromas, and malignant peripheral nerve sheath tumors, MUM1 is detected in only one in eight cases of spindle cell and desmoplastic melanomas. With the exception of desmoplastic and spindle cell melanomas, MUM1 appears to be a sensitive and specific immunohistochemical stain for melanocytic lesions and may prove to be a useful addition to the current panel of melanoma markers.

\footnotetext{
Copyright () 2003 by The United States and Canadian Academy of Pathology, Inc.

VOL. 16, NO. 8, P. 802, 2003 Printed in the U.S.A.

Date of acceptance: May 1, 2003.

Address reprint requests to: Uma Sundram, M.D., Ph.D., Department of Pathology, Stanford University Medical Center, 300 Pasteur Drive, Room H2110, Stanford, CA 94305; fax: 650-725-7409; e-mail: sundram @stanford.edu.

DOI: $10.1097 / 01 . M P .0000081726 .49886 . C F$
}

KEY WORDS: HMB45, MelanA, Melanocytic lesions, MUM1, S100.

Mod Pathol 2003;16(8):802-810

The diagnosis of metastatic malignant melanoma is one of the most difficult in surgical pathology. The pattern of spread of melanoma can be extremely variable, and the histologic characteristics are such that melanoma may mimic many other benign and malignant entities. Frequently, immunohistochemical confirmation is necessary to make a definitive diagnosis of metastatic melanoma. The immunohistochemical markers antiS100, anti-gp100 (HMB45), and anti-MelanA have been used widely in clinical settings to establish the diagnosis of melanoma. Unfortunately, each marker is hampered by lack of specificity and/or sensitivity. For example, anti-S100, the most sensitive of the three, lacks specificity as it stains a variety of other neoplasms (1-3). Although HMB45 and anti-MelanA are both more specific markers for melanoma, they are less sensitive, and staining is often patchy and dependent on technique $(4-7,19)$.

The MUM1 (multiple myeloma 1)/IRF4 (interferon regulatory factor 4; also known as LSIRF, Pip, and ICSAT) gene product is a member of the interferon regulatory factor family of transcription factors $(8-11)$. These factors are known to play an important role in the regulation of gene expression in response to interferon and other cytokines. In multiple myeloma, MUM1 is activated at the transcriptional level by the chromosomal translocation $\mathrm{t}(6 ; 14)(\mathrm{p} 25 ; \mathrm{q} 32)$, which places the transcription factor next to the IgH enhancer locus (12). The oncogenic properties of MUM1 in hematopoietic processes have been attributed to this translocation and the consequent overexpression of the gene. In addition, studies with MUM1 - / - mice have shown that the mice are dysfunctional in both B- and T-cell-mediated immune processes, supporting the notion that expression of MUM1 is essential for 
proper function of mature B cells and cytotoxic $\mathrm{T}$ cells (13).

Although the role of MUM1 in hematolymphoid malignancies and immune regulation is recognized, a detailed analysis of the expression patterns of the MUM1 protein in other tissues has only recently been initiated $(14,15)$. Elsewhere, we characterized the expression of the MUM1 protein in a wide variety of hematolymphoid malignancies, nonhematolymphoid malignancies, and normal tissues, using a murine monoclonal antibody (MUM1p) and tissue array techniques (16). Our results demonstrated that the MUM1 protein can be detected in neoplasms of plasmacytic differentiation as well as in a wide variety of B-, T-, and NK-cell lymphomas. In addition, although the MUM1 protein was not found to be expressed in the other malignant neoplasms tested, 5 of 22 melanomas showed strong nuclear staining. This is consistent with results obtained during initial cloning and characterization of the hLSIRF mRNA (which encodes the MUM1 transcription factor), in which expression of the mRNA was detected in a melanoma cell line as well as in normal melanocytes (8).

In this study, we have further characterized the expression pattern of the MUM1 protein in 61 melanocytic lesions. We have also compared the sensitivity and specificity of the murine antibody MUM1 with anti S100, HMB45, and anti-MelanA. Finally, we have examined the ability of the MUM1 antibody to distinguish nerve sheath proliferations such as schwannomas, neurofibromas, and malignant peripheral nerve sheath tumors (MPNST) from melanocytic proliferations, as nerve sheath lesions are frequently in the differential diagnosis of benign melanocytic lesions and desmoplastic malignant melanoma (20).

\section{MATERIALS AND METHODS}

\section{Case Selection}

A total of 61 melanocytic cases and 10 nerve sheath cases seen at Stanford University Medical Center comprise this study and were selected on the basis of the original reported diagnosis. The melanocytic cases include 22 primary malignant melanomas, 3 spindle cell melanomas, 5 desmo- plastic malignant melanomas, 14 metastatic melanomas, 15 benign nevi, and 2 clear cell sarcomas (malignant melanoma of soft parts). The metastatic melanomas include four lesions involving the brain; three involving the soft tissues; two involving the lung; and one each involving the parotid, spleen, live, pleura, and lymph node. The nerve sheath tumors include four neurofibromas, three schwannomas, and three malignant peripheral nerve sheath tumors (MPNST). Hematoxylin and eosin-stained sections together with immunohistologic studies (if performed at the time of diagnosis) of each case were reviewed to confirm the original findings. All cases were selected before immunohistochemical staining with the antibody for MUM1. Immunohistochemical staining with the antibodies for S100, HMB45, and MelanA was then added if not performed as part of the original analysis. A subset of 10 benign nevi (7 Spitz nevi and 3 intradermal melanocytic nevi) was tested only with the antibodies for MUM1 and S100.

\section{Immunohistochemistry}

Immunohistochemical studies for all markers were performed on conventional sections of formalin-fixed and paraffin-embedded tissue using a modified avidin-biotin peroxidase complex (ABC) amplification and detection system (17). Primary antibodies were directed against MUM1 (14), S100 protein (DAKO, Carpinteria, CA), gp100 (HMB45, DAKO), and MelanA protein (clone A103, DAKO). The antibody sources, dilutions, and enzymatic- or microwave based epitope retrieval procedures used before incubation with the primary antibody are listed in Table 1. Serial sections of $4 \mu \mathrm{m}$ were cut from paraffin blocks and deparaffinized in xylene and hydrated in a graded series of alcohol. Endogenous peroxidase was suppressed by incubation with $3 \% \mathrm{H}_{2} \mathrm{O}_{2}$. All primary antibodies were incubated for 30 minutes, followed by secondary antibody incubation (40 minutes) and the combination of streptavidin with biotinylated peroxidase (40 minutes). Heat-induced antigen retrieval was carried out by microwave pretreatment in EDTA (1 $\mathrm{mm}, \mathrm{pH}$ 8.0) for 15 minutes, followed by a 30minute cooling period before staining with the an-

TABLE 1. Monoclonal Antibodies, Their Sources and Dilutions, and the Pretreatment Modalities Used in This Study

\begin{tabular}{llccl}
\hline Antigen & Clone & Pretreatment & $\begin{array}{c}\text { Antibody } \\
\text { Dilution }\end{array}$ & Source \\
\hline S100 & Polyclonal & None & $1: 4000$ & Dako, Carpinteria, CA \\
HMB45 & & None & $1: 40$ & Dako \\
MelanA & A103 & MW & $1: 50$ & Dako \\
MUM1 & Monoclonal & MW & $1: 4$ & BF \\
MUM1 (E) & Monoclonal & MW & $1: 50$ & BF \\
\hline
\end{tabular}

$\mathrm{MW}=$ microwave heating adjusted to near-boiling in EDTA-buffer, 15 minutes at $\mathrm{pH}$ 8.0, followed by a 30-minute cooling period; $\mathrm{E}=\mathrm{En}$ Vision+ system; BF $=$ B, Falini, Perugia University (14). 
tibodies for MUM1 and MelanA. The immunohistochemical analysis for expression of the MUM1 protein was optimized in previously performed experiments (16). To reduce background nonspecific staining, sections of selected cases of desmoplastic malignant melanoma were incubated with the MUM1 antibody, followed by analysis using the EnVision+ System (DAKO), which uses neither streptavidin nor biotin for detection purposes.

Staining for the MUM1 protein was localized predominantly to the nucleus, although weak to moderate cytoplasmic staining was also present in most cases with nuclear positivity (15-16). Nuclear staining alone or in combination with cytoplasmic staining was considered positive for anti-S100, whereas cytoplasmic staining alone was considered negative (21). Cytoplasmic staining alone was considered positive for HMB45 and anti-MelanA. Staining for all markers was reported in the following categories: strongly positive $=$ moderate to intense staining of $\geq 20 \%$ of lesional cells; weakly positive = faint, moderate, or intense staining of $\geq 5 \%$ but $<20 \%$ of lesional cells; and negative $=$ faint or moderate staining of $<5 \%$ to no staining of lesional cells. Positive scoring constituted the sum of strong and weak staining as defined above. In biopsies of skin, normal basal layer melanocytes served as positive internal controls, and lymphocytes and plasma cells served as positive internal controls in other samples for anti-MUM1. Epithelial cells in tonsillar tissue were employed as a negative external control.

\section{RESULTS}

\section{MUM1 Staining in Conventional Primary and Metastatic Melanomas}

The results of immunohistochemical studies with the antibodies for MUM1, S100, HMB45, and MelanA are summarized in Table 2 and shown in Figure 1.

Staining for the MUM1 protein was positive in $31 / 36(92 \%)$ cases of melanoma (21 of 22 conventional primary melanomas (95\%) and 12 of 14 metastatic melanomas $(86 \%)$ ). This trend is similar to the staining pattern of anti-S100, which was positive in all melanomas tested (100\%). In contrast, HMB45 was positive in 17 cases of conventional primary melanoma $(77 \%)$ and 11 cases of metastatic melanoma (79\%; positive staining in all melanomas, $78 \%$ ). Anti-MelanA was positive in 19 cases of conventional primary melanoma $(86 \%)$ and 8 cases of metastatic melanoma $(50 \%$; positive staining in all melanomas, $75 \%$ ).

If only strong positive staining is taken into account, MUM1 staining was found to be strongly positive in $86 \%$ of conventional primary melanomas (19/22) and $78 \%$ of metastatic melanomas (11/

TABLE 2. Analysis of the Antibodies for MUM1, S100, gp100 (HMB45), and MelanA in Melanocytic Lesions

\begin{tabular}{|c|c|c|c|c|c|c|c|c|c|c|c|c|c|c|c|c|c|c|c|c|c|}
\hline \multirow{2}{*}{ Diagnosis } & \multirow{2}{*}{$n$} & \multicolumn{5}{|c|}{ MUM1 } & \multicolumn{5}{|c|}{ S100 } & \multicolumn{5}{|c|}{ HMB45 } & \multicolumn{5}{|c|}{ MelanA } \\
\hline & & $>20 \%$ & $<20 \%$ & 0 & $\% \mathrm{P}$ & $\% \mathrm{SP}$ & $>20 \%$ & $<20 \%$ & 0 & $\% \mathrm{P}$ & $\% \mathrm{SP}$ & $>20 \%$ & $<20 \%$ & 0 & $\% \mathrm{P}$ & $\% \mathrm{SP}$ & $>20 \%$ & $<20 \%$ & 0 & $\% \mathrm{P}$ & $\% \mathrm{SP}$ \\
\hline Prim melanoma, MIS & 22 & 19 & 2 & 1 & 95 & 86 & 19 & 3 & 0 & 100 & 86 & 16 & 1 & 5 & 77 & 73 & 15 & 4 & 3 & 86 & 68 \\
\hline Met melanoma (total) & 14 & 11 & 1 & 2 & 86 & 78 & 13 & 1 & 0 & 100 & 93 & 11 & 0 & 3 & 78 & 78 & 5 & 3 & 6 & 57 & 36 \\
\hline Brain & 4 & 4 & 0 & 0 & 100 & 100 & 4 & 0 & 0 & 100 & 100 & 4 & 0 & 0 & 100 & 100 & 2 & 2 & 0 & 100 & 50 \\
\hline Parotid & 1 & 1 & 0 & 0 & 100 & 100 & 1 & 0 & 0 & 100 & 100 & 1 & 0 & 0 & 100 & 100 & 1 & 0 & 0 & 100 & 100 \\
\hline Spleen & 1 & 1 & 0 & 0 & 100 & 100 & 1 & 0 & 0 & 100 & 100 & 1 & 0 & 0 & 100 & 100 & 0 & 0 & 1 & 0 & 0 \\
\hline Soft tissue & 3 & 2 & 0 & 1 & 67 & 67 & 3 & 0 & 0 & 100 & 100 & 1 & 0 & 2 & 33 & 33 & 0 & 1 & 2 & 33 & 0 \\
\hline Liver & 1 & 1 & 0 & 0 & 100 & 100 & 0 & 1 & 0 & 100 & 0 & 1 & 0 & 0 & 100 & 100 & 1 & 0 & 0 & 100 & 100 \\
\hline Lung & 2 & 1 & 1 & 0 & 100 & 50 & 2 & 0 & 0 & 100 & 100 & 2 & 0 & 0 & 100 & 100 & 0 & 0 & 2 & 0 & 0 \\
\hline Pleura & 1 & 1 & 0 & 0 & 100 & 100 & 1 & 0 & 0 & 100 & 100 & 1 & 0 & 0 & 100 & 100 & 1 & 0 & 0 & 100 & 100 \\
\hline Lymph node & 1 & 0 & 0 & 1 & 0 & 0 & 1 & 0 & 0 & 100 & 100 & 0 & 0 & 1 & 0 & 0 & 0 & 0 & 1 & 0 & 0 \\
\hline Prim + met & 36 & 30 & 3 & 3 & 92 & 83 & 32 & 4 & 0 & 100 & 89 & 27 & 1 & 8 & 78 & 75 & 20 & 7 & 9 & 75 & 56 \\
\hline SCM & 3 & 1 & 1 & 1 & 67 & 33 & 2 & 1 & 0 & 100 & 66 & 1 & 1 & 1 & 67 & 33 & 1 & 0 & 2 & 33 & 33 \\
\hline DM & 5 & 0 & 0 & 5 & 0 & 0 & 5 & 0 & 0 & 100 & 100 & 0 & 0 & 5 & 0 & 0 & 0 & 0 & 5 & 0 & 0 \\
\hline CCS & 2 & 2 & 0 & 0 & 100 & 100 & 2 & 0 & 0 & 100 & 100 & 1 & 1 & 0 & 100 & 50 & 0 & 1 & 1 & 50 & 0 \\
\hline IDN & 1 & 1 & 0 & 0 & 100 & 100 & 1 & 0 & 0 & 100 & 100 & 0 & 0 & 1 & 0 & 0 & 0 & 1 & 0 & 100 & 0 \\
\hline IDN* & 3 & 2 & 1 & 0 & 100 & 67 & 3 & 0 & 0 & 100 & 100 & $\mathrm{~N}$ & $\mathrm{~N}$ & $\mathrm{~N}$ & $\mathrm{~N}$ & $\mathrm{~N}$ & $\mathrm{~N}$ & $\mathrm{~N}$ & $\mathrm{~N}$ & $\mathrm{~N}$ & $\mathrm{~N}$ \\
\hline $\mathrm{CMN}$ & 2 & 2 & 0 & 0 & 100 & 100 & 2 & 0 & 0 & 100 & 100 & 1 & 1 & 0 & 100 & 50 & 2 & 0 & 0 & 100 & 100 \\
\hline Congenital nevi & 2 & 2 & 0 & 0 & 100 & 100 & 2 & 0 & 0 & 100 & 100 & 0 & 2 & 0 & 100 & 0 & 2 & 0 & 0 & 100 & 100 \\
\hline Spitz & 3 & 1 & 0 & 2 & 33 & 33 & 3 & 0 & 0 & 100 & 100 & 0 & 1 & 2 & 33 & 0 & 1 & 0 & 2 & 33 & 33 \\
\hline Spitz* & 7 & 7 & 0 & 0 & 100 & 100 & 7 & 0 & 0 & 100 & 100 & $\mathrm{~N}$ & $\mathrm{~N}$ & $\mathrm{~N}$ & $\mathrm{~N}$ & $\mathrm{~N}$ & $\mathrm{~N}$ & $\mathrm{~N}$ & $\mathrm{~N}$ & $\mathrm{~N}$ & $\mathrm{~N}$ \\
\hline Total nevi & 18 & 15 & 1 & 2 & 89 & 83 & 11 & 0 & 0 & 100 & 100 & 1 & 4 & 3 & 63 & 13 & 5 & 1 & 2 & 75 & 63 \\
\hline Neurofibroma & 4 & 0 & 0 & 4 & 0 & 0 & 1 & 3 & 0 & 100 & 33 & $\mathrm{~N}$ & $\mathrm{~N}$ & $\mathrm{~N}$ & $\mathrm{~N}$ & $\mathrm{~N}$ & $\mathrm{~N}$ & $\mathrm{~N}$ & $\mathrm{~N}$ & $\mathrm{~N}$ & $\mathrm{~N}$ \\
\hline Schwannoma & 3 & 0 & 0 & 3 & 0 & 0 & 3 & 0 & 0 & 100 & 100 & $\mathrm{~N}$ & $\mathrm{~N}$ & $\mathrm{~N}$ & $\mathrm{~N}$ & $\mathrm{~N}$ & $\mathrm{~N}$ & $\mathrm{~N}$ & $\mathrm{~N}$ & $\mathrm{~N}$ & $\mathrm{~N}$ \\
\hline MPNST & 3 & 0 & 0 & 3 & 0 & 0 & 3 & 0 & 0 & 100 & 100 & $\mathrm{~N}$ & $\mathrm{~N}$ & $\mathrm{~N}$ & $\mathrm{~N}$ & $\mathrm{~N}$ & $\mathrm{~N}$ & $\mathrm{~N}$ & $\mathrm{~N}$ & $\mathrm{~N}$ & $\mathrm{~N}$ \\
\hline
\end{tabular}

$n=$ Number of cases; Prim = primary; Met = metastatic; MIS = melanoma in situ; SCM = spindle cell melanoma; DM = Desmoplastic melanoma; CCS = Clear cell sarcoma; IDN = intradermal melanocytic nevi; IDN* = intradermal melanocytic nevi tested only with MUM1 and S100; CMN = compound melanocytic nevi; Spitz = Spitz nevi; Spitz* = Spitz nevi tested only with MUM1 and S100; N = not tested. Immunohistochemical staining for MUM1, S100, gp100 (HMB45), and MelanA proteins was scored as follows: $>20 \%$ (strongly positive) $=$ moderate to intense staining of at least $20 \%$ of lesional cells; $<20 \%$ (weakly positive) = faint, moderate, or intense staining of at least $5 \%$ but less than $20 \%$ of lesional cells; 0 (negative) $=$ faint or moderate staining of less than $5 \%$ to no staining of lesional cells; SP = percent strongly positive; $\mathrm{P}=$ percent positive. Nuclear or nuclear and cytoplasmic staining was scored as positive with the antibodies for MUM1 and S100, while cytoplasmic staining was scored as positive with HMB45 and anti-MelanA. 

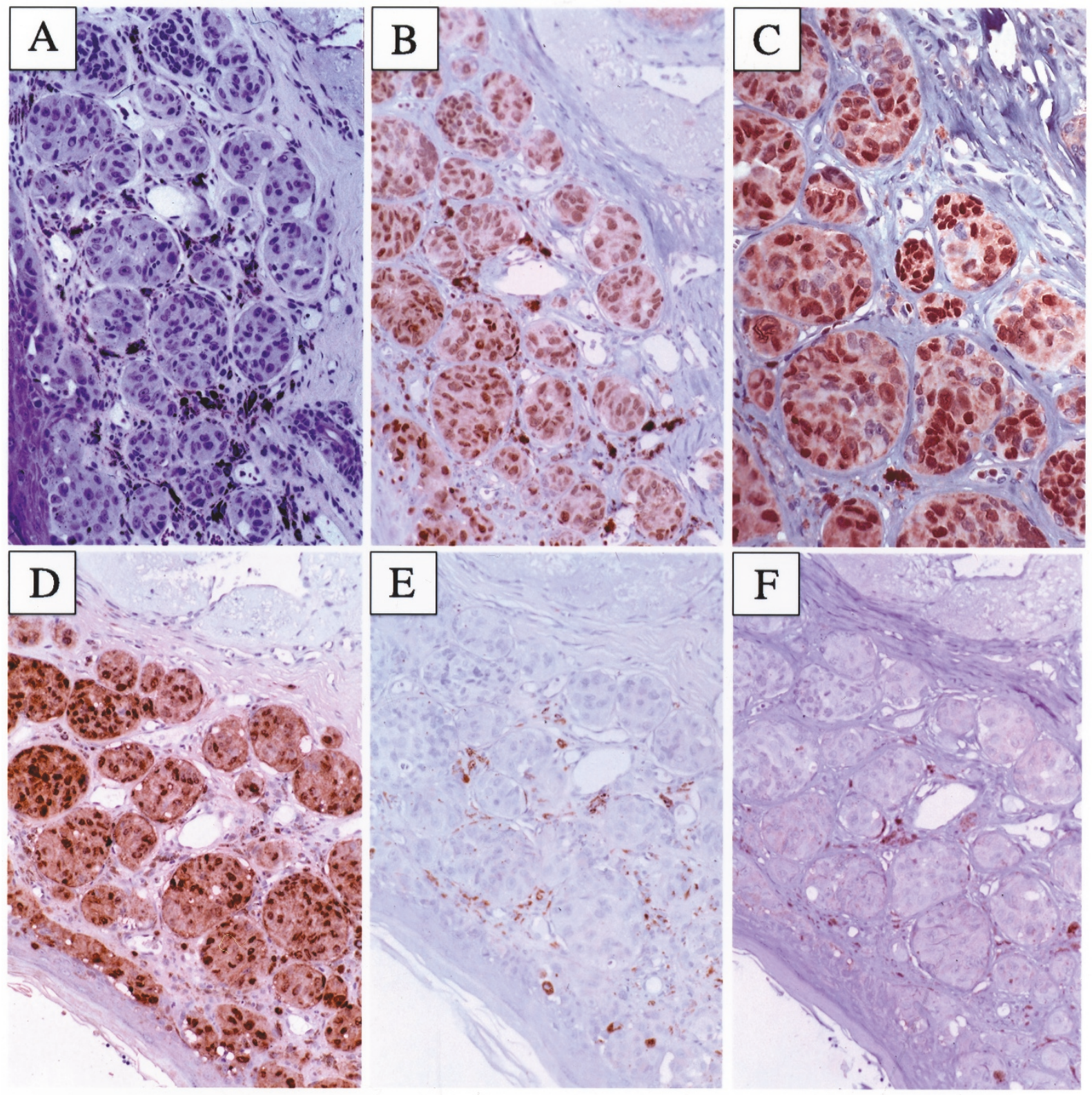

FIGURE 1. Immunohistochemical studies on conventional tissue sections of primary melanoma. A, hematoxylin and eosin- $(\mathrm{H}+\mathrm{E})$ stained section with clusters of melanoma cells and pigment within melanophages (magnification, 100×). B-C, MUM1 is strongly expressed in nuclei with weak to moderate cytoplasmic staining (magnification, $100 \times$ and $200 \times$, respectively). D, S100 is also strongly expressed in nuclei with moderate cytoplasmic staining (magnification, 100×). E, HMB45 and F, MelanA staining are not present in any of the lesional cells (magnification, 100×).

14; strong positivity in all melanomas, $83 \%)$. Similarly, S100 was strongly positive in 19 of 22 conventional primary melanomas (86\%) and 13 of 14 metastatic melanomas $(93 \%$; overall strong positivity, 89\%). In contrast, HMB45 was strongly positive in 16 cases of conventional primary melanoma (73\%) and 11 cases of metastatic melanoma $(79 \%$; overall strong positivity, $75 \%$ ). Anti-MelanA was strongly positive in 15 cases of conventional primary melanoma $(68 \%)$ and 5 cases of metastatic melanoma (36\%; overall strong positivity, 56\%).

Anti-MUM1 demonstrated strong positivity in four cases of conventional melanomas that were negative (faint or moderate staining of $<5 \%$ to no staining of lesional cells) or weakly positive (faint, moderate, or intense staining of $\geq 5 \%$ but $<20 \%$ of lesional cells) with HMB45 and anti-MelanA. Four additional cases that were weakly positive with anti-S100 and six additional cases that were negative or weakly positive with anti-MelanA alone were also strongly positive with anti-MUM1. Of note, $2 / 2$ cases of clear cell sarcoma (malignant melanoma of soft parts) were strongly positive with the antibodies to both MUM1 and S100, whereas only one case was strongly positive with HMB45, and neither was strongly positive with anti-MelanA. 


\section{MUM1 Staining in Spindle Cell and Desmoplastic Melanomas}

Spindle cell melanomas are defined as malignant melanocytic tumors primarily composed of neoplastic spindled cells with no significant desmoplasia. In contrast, primary and metastatic desmoplas- tic melanomas contain narrow fascicles of spindled pleomorphic neoplastic cells in a prominent collageneous stroma (Fig. 2). Anti-MUM1 strongly highlighted one of three cases of spindled cell melanoma, similar to HMB45 and anti-MelanA, whereas anti-S100 strongly highlighted two of three cases.
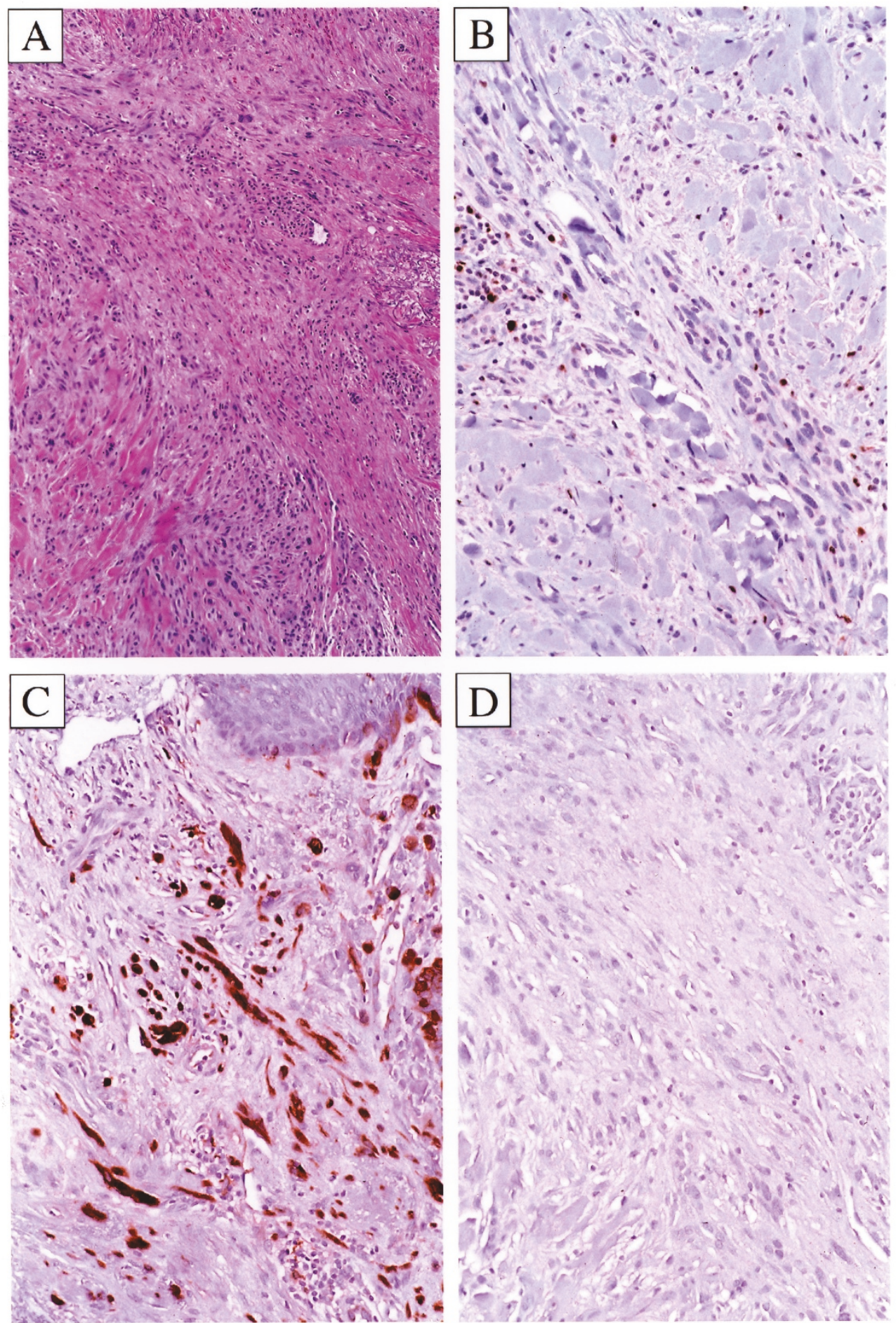

FIGURE 2. Immunohistochemical studies on conventional tissue sections of desmoplastic melanoma. A, hematoxylin and eosin-stained section shows atypical spindled cells infiltrating a collagenous stroma. B, MUM1 staining is present in small lymphocytes within the accompanying inflammatory infiltrate but is present in $<5 \%$ of lesional cells. C, S100 shows strong nuclear staining with weak cytoplasmic staining; D, HMB45 staining is present in $<5 \%$ of lesional cells (magnification, $100 \times$ ). 
The MUM1 antibody showed faint cytoplasmic but no nuclear staining in two of five cases of desmoplastic melanoma. However, cytoplasmic staining was abolished by the use of the EnVision+ detection system, which suggests that the staining may be secondary to residual endogenous avidin binding. The overall staining pattern is similar to that seen with HMB45 and anti-MelanA, which failed to highlight any of the five cases of desmoplastic melanoma.

\section{MUM1 staining in Benign Nevi}

Eighteen cases of benign nevi (intradermal melanocytic nevi, compound melanocytic nevi, and Spitz nevi) were tested with anti-MUM1 and compared with the staining patterns of anti-S100, HMB45, and anti-MelanA (a subset of three intradermal melanocytic nevi and seven Spitz nevi were not tested with antibodies for HMB45 and MelanA, see Table 2). Strong positivity was seen with antiMUM1 in 3 cases of intradermal melanocytic nevi, all 4 cases of compound melanocytic nevi (including congenital type nevi), and 8 of 10 cases of Spitz nevi. In the compound nevi, anti-MUM1 stained both junctional and intradermal melanocytic components with equivalent intensity. In contrast, HMB45 was strongly positive in only one case of compound melanocytic nevus and weakly stained or failed to stain all other nevi (especially the intradermal component). Anti-MelanA was strongly positive in all cases of compound melanocytic nevi but was either weak or negative on all other nevi tested.

\section{MUM1 Staining in Nerve Sheath Neoplasms}

To study the ability of the MUM1 antibody to distinguish between nerve sheath and melanocytic lesions, 10 nerve sheath lesions (4 neurofibromas, 3 schwannomas, and 3 MPNST) were tested with anti-MUM1 and anti-S100, and the results were compared with staining of intradermal melanocytic nevi (Fig. 3). Anti-MUM1 was strongly positive in three of four cases of intradermal melanocytic nevi but did not stain any of the nerve sheath lesions. Anti-S100 was strongly positive in all three cases of schwannoma and MPNST, respectively, and in one of four cases of neurofibroma. Finally, a neurofibroma that also had features of a neurotized intradermal melanocytic nevus was strongly highlighted by the S100 antibody but not by anti-MUM1, HMB45, or anti-MelanA.

\section{DISCUSSION}

Although the early studies of MUM1 offered evidence that this molecule may play a significant role in terminal B-cell differentiation and hence be a potentially specific marker for plasmacytic differentiation, studies in our laboratories (16) and others $(8,10,11,14,15)$ have demonstrated that the expression of MUM1 is not limited to plasma cells. However, examination of expression of MUM1 in a large number and variety of human malignancies has shown that MUM1 is present in a wide spectrum of hematolymphoid neoplasms and in malignant melanomas but is absent in the other human neoplasms tested (16). The discovery of MUM1 expression in melanocytic lesions substantiates previous observations made during initial cloning and characterization of the hLSIRF mRNA encoding the MUM1 transcription factor, which showed that mRNA expression of MUM1 was noted in a melanoma cell line and in normal melanocytes (8).

In the current study addressing the sensitivity of the MUM1 antibody, we found that it highlighted the majority of melanomas, with $92 \%$ of conventional primary and metastatic melanomas showing positive staining (83\% strong staining). These findings confirm prior results that demonstrated the potential of MUM1 to stain melanocytic lesions, although the prior studies may have underestimated its sensitivity (as they were performed on tissue microarrays that may not have accounted for tumor heterogeneity; 16). In addition, MUM1 proved to be a more sensitive marker than either HMB45 or anti-MelanA in cases of conventional primary and metastatic melanomas. The MUM1 antibody showed strong positivity in four cases of melanoma that failed to stain or were weakly positive with HMB45 and six additional cases that failed to stain with anti-MelanA. These results demonstrate the ability of this antibody to detect melanomas which could be missed if certain conventional melanocytic markers are employed. Moreover, four additional cases that were weakly positive with anti-S100 were strongly positive with anti-MUM1. Although only two were studied, the cases of clear cell sarcoma had interesting results, as these were strongly and uniformly highlighted by both anti-MUM1 and anti-S100, but only one case demonstrated strong positive staining by HMB45 and neither was strongly positive with anti-MelanA. The MUM1 antibody also showed more diffuse and strong staining of benign melanocytic nevi than either HMB45 or antiMelanA, strongly staining $75 \%$ of benign nevi in this study, compared with $13 \%$ and $63 \%$, respectively. When staining of Spitz nevi is examined, MUM1 strongly stains $80 \%$ of nevi $(8 / 10)$, which compares favorably with the marker for S100 (100\%). The MUM1 antibody did not stain any of the nerve sheath lesions in our study, which included neurofibromas, schwannomas, and MPNST. As MUM1 expression appears to be present in both 

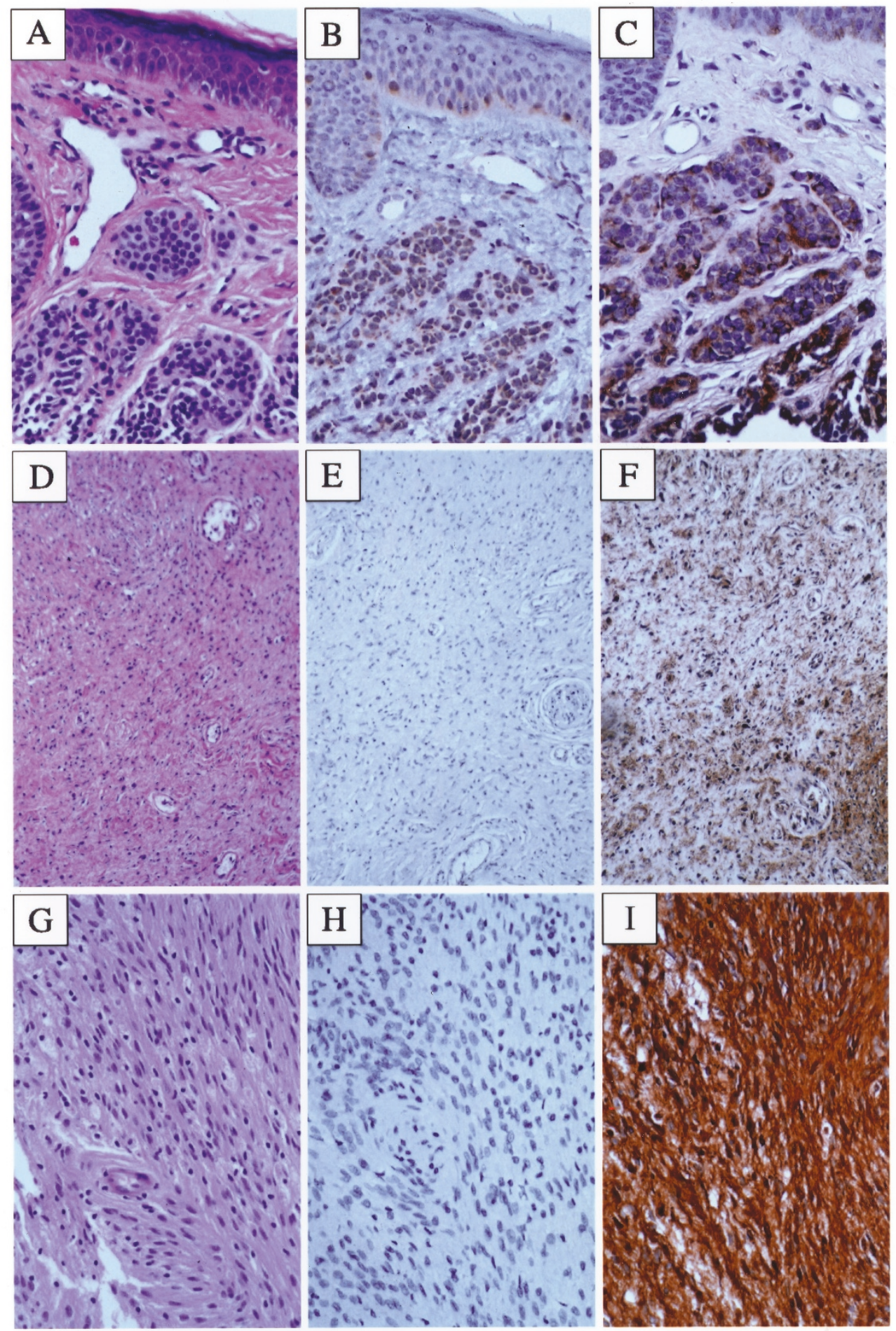

FIGURE 3. Immunohistochemical studies analysis on conventional tissue sections of intradermal nevus, neurofibroma, and schwannoma: A, hematoxylin and eosin $(\mathrm{H}+\mathrm{E})$-stained section of intradermal nevus shows nests of bland nevus cells within the dermis (magnification, 100 $\times$ ); B-C, MUM1 and S100 staining are strongly positive in the nucleus, with weak cytoplasmic staining (magnification, $100 \times$ ). D, $\mathrm{H}+\mathrm{E}-\mathrm{stained}$ section of neurofibroma shows a paucicellular population of stellate cells within a myxoid stroma that encircle nerves (magnification, $40 \times$ ). E, MUM1 does not highlight any lesional cells (magnification, $40 \times$ ). F, S100 staining is localized to the nuclei of the stellate cells (magnification, $40 \times$ ). G, $\mathrm{H}+\mathrm{E}$-stained section of schwannoma shows a population of wavy spindled cells (magnification, 100×). H, MUM1 does not highlight lesional cells (magnification, $100 \times$ ). I, S100 strongly stains both nuclei and cytoplasm (magnification, $100 \times$ ).

benign nevi and in melanomas, the role of this protein in the biologic behavior of primary cutaneous pigmented lesions is unclear and may need additional studies for clarification.
From a prior study in our laboratory with tissue microarrays, it is clear that the MUM1 antibody has great specificity for hematolymphoid and melanocytic tumors (16). In that study, 944 nonhema- 
tolymphoid neoplasms and normal tissues were examined, including those of the breast, adrenal, colon, lung, kidney, and skin, and MUM1 expression was detected only in melanomas. In the current study, we have chosen to compare the results at the level of strong staining in particular (defined as moderate to intense staining of $\geq 20 \%$ of lesional cells). Weak staining results (defined as faint, moderate, or intense staining of $\geq 5 \%$ but $<20 \%$ of lesional cells) are of potentially less value diagnostically as they merge into equivocal levels of staining. In addition, intense but focal staining may lead to false-negative evaluations in small biopsy specimens. Emphasis on the results of strong staining may account for the discrepancy in the sensitivities reported in this study for conventional melanocytic markers such as S100, HMB45, and MelanA and those reported in the literature.

The use of immunohistochemistry in the diagnosis of melanoma has been manifold, but primarily in the analysis of primary and metastatic epithelioid or spindle cell lesions of unknown type and in the examination of sentinel nodes for metastatic melanoma. As the presence of melanocytic differentiation is clear in most cases of primary epithelioid melanomas, immunohistochemistry is usually not employed. However, in rare cases of primary epithelioid melanomas and in most cases of metastatic melanomas, the presence of melanocytic differentiation is not morphologically obvious, and a panel of melanocytic and epithelial markers is used to establish the diagnosis. The major advantage of the MUM1 antibody is in these cases, as antiMUM1 showed strong positive staining in a higher percentage of epithelioid melanocytic lesions than either HMB45 or anti-MelanA, and greater specificity against epithelioid lesions than S100.

Spindle cell melanomas are usually considered in the differential diagnosis of spindle cell lesions of the skin and are frequently highlighted by both anti-S100 and HMB45, which establishes their origin from melanocytes. On the other hand, desmoplastic melanomas are composed of atypical spindled cells embedded in bundles of collagenous stroma, and these lesions are frequently stained only by anti-S100 (18). As anti-MUM1 strongly highlighted only $33 \%(1 / 3)$ of spindle cell melanomas, whereas anti-S100 strongly stained twice as many cases $(2 / 3,66 \%)$, the MUM1 antibody is not as useful as anti-S100 in these cases. In addition, similar to the cases of HMB45 and anti-MelanA, anti-MUM1 did not highlight any cases of desmoplastic melanoma. However, the numbers of cases of both spindle cell and desmoplastic melanoma are small, and a larger number may be necessary to substantiate these preliminary results.

Finally, a melanocytic panel is frequently used in the assessment of sentinel lymph nodes for meta- static melanoma. The MUM1 antibody is at a significant disadvantage in these situations compared with the other melanocytic markers as it reacts with lymphocytes and plasma cells. However, the ability to stain both lymphocytes and melanocytes allows for the presence of readily identifiable positive internal controls in other sites.

In summary, the B-cell proliferation/differentiation protein MUM1 is a sensitive and specific marker for conventional primary and metastatic melanomas and benign melanocytic nevi. The current study demonstrates that when screening for strong positive staining, the MUM1 antibody is more sensitive for melanocytic lesions than HMB45 and anti-MelanA but not as sensitive as anti-S100 (although it does show better specificity than antiS100). As such, MUM1 is a helpful reagent for use in diagnostic panels when the diagnosis of melanoma is under consideration. However, MUM1 may not be as useful as the conventional markers for melanoma in the context of the sentinel lymph node, as this protein is expressed in both melanocytes and lymphocytes.

Acknowledgments: The authors thank Dr. Brunangelo Falini (Department of Hematology, Perugia University, Italy) for use of the MUM1 antibody (MUM1p) and thank Elizabeth Domanay for technical assistance.

\section{REFERENCES}

1. Moore BW. A soluble protein characteristic of the nervous system. Biochem Biophys Res Commun 1965;19:739-44.

2. Vanstapel MJ, Gatter KC, Wolf-Peeters C, Mason DY, Desmet VD. New site of human S100 immunoreactivity detected with monoclonal antibodies. Am J Clin Pathol 1986;85:160-8.

3. Takahashi $\mathrm{K}$, Isobe $\mathrm{T}$, Ohtsuki $\mathrm{Y}$, Akagi $\mathrm{T}$, Sonobe $\mathrm{H}$, Okuyama T. Immunohistochemical study on the distribution of alpha and beta subunits of S100 protein in human neoplasms and normal tissues. Virchows Arch (B) 1984;45: 385-96.

4. Kaufmann O, Koch S, Burghard J, Audring H, Dietel M. Tyrosinase, Melan A, and KBA62 as markers for the immunohistochemical identification of metastatic amelanotic melanomas on paraffin sections. Mod Pathol 1998;11:740-6.

5. Busam K, Chen Y, Old L, Stockert E, Iversen K, Copeland KA, et al. Expression of MelanA (MART 1) in benign melanocytic nevi and primary cutaneous malignant melanoma. Am J Surg Pathol 1998;22:976-82.

6. Skelton H, Maceira J, Smith K, McCarthy W, Lupton G, Graham J. HMB45 negative spindle cell melanoma. Am J Dermatopathol 1997;19:580-4.

7. Gown A, Vogel A, Hoak A, Gough F, NcNutt M. Monoclonal antibodies specific for melanocytic tumors distinguish subpopulations from melanocytes. Am J Pathol 1986;123:195203.

8. Grossman A, Mittrucker HW, Nicholl J, Suzuki A, Chung S, Antonio L, et al. Cloning of human lymphocyte-specific interferon regulatory factor (hLSIRF/hIRF4) and mapping of the gene to 6p23-p25. Genomics 1996;37:229-33. 
9. Eisenbeis CF, Singh H, Storb U. Pip, a novel IRF family member, is a lymphoid-specific, PU.1-dependent transcriptional activator. Genes Dev 1995;9:1377-87.

10. Matsuyama T, Grossman A, Mittrucker HW, Siderovski DP, Kiefer F, Kawakami T, et al. Molecular cloning of LSIRF, a lymphoid-specific member of the interferon regulatory factor family that binds the interferon- stimulated response element (ISRE). Nucleic Acids Res 1995;23:2127-36.

11. Yamagata T, Nishida J, Tanaka S, Sakai R, Mitani K, Yoshida $\mathrm{M}$, et al. A novel interferon regulatory factor family transcription factor, ICSAT/Pip/LSIRF, that negatively regulates the activity of interferon- regulated genes. Mol Cell Biol 1996;16: 1283-94.

12. Iida S, Rao PH, Butler M, Corradini P, Boccadoro M, Klein B, et al. Deregulation of MUM1/IRF4 by chromosomal translocation in multiple myeloma. Nat Genet 1997;17:226-30.

13. Mittrucker HW, Matsuyama T, Grossman A, Kundig TM, Potter J, Shahinian A, et al. Requirement for the transcription factor LSIRF/IRF4 for mature B and T lymphocyte function. Science 1997;275:540-3.

14. Falini B, Fizzotti M, Pucciarini A, Bigerna B, Marafioti T, Gambacorta M, et al. A monoclonal antibody (MUM1p) detects expression of the MUM1/IRF4 protein in a subset of germinal center B cells, plasma cells, and activated T cells. Blood 2000;95:2084-92.
15. Tsuboi K, Iida S, Inagaki H, Kato M, Hayami Y, Hanamura I, et al. MUM1/IRF4 expression as a frequent event in mature lymphoid malignancies. Leukemia 2000;14:449-56.

16. Natkunam Y, Warnke RA, Montgomery K, Falini B, van de Rijn M. Analysis of MUM1/IRF4 protein expression utilizing tissue microarrays and immunohistochemistry. Mod Pathol 2001;14:686-94.

17. Bindl JM, Warnke RA. Advantages of detecting monoclonal antibody binding to tissue sections with biotin and avidin reagents in Coplin jars. Am J Clin Pathol 1986;85:490-3.

18. Longacre TA, Egbert BM, Rouse RV. Desmoplastic and spindle cell malignant melanoma: an immunohistochemical study. Am J Surg Pathol 1996;20:1489-500.

19. Orosz Z. MelanA/Mart 1 expression in various melanocytic lesions and in non melanocytic soft tissue tumours. Histopathology 1999;34:517-25.

20. Gray MH, Smoller BR, McNutt NS, Hsu A. Immunohistochemical demonstration of factor XIIIa expression in neurofibromas: a practical means of differentiating these tumors from neurotized melanocytic nevi and schwannomas. Arch Dermatol 1999;126:472-6.

21. Wick MR. Immunohistologic features of melanocytic neoplasms. In: Dabbs DJ, editor. Diagnostic immunohistochemistry. 1st ed. New York: Churchill Livingstone; 2002. p. 150. 